\title{
The similarity of the interstellar comet 21/Borisov to Solar System comets from high-resolution optical spectroscopy
}

\author{
C. Opitom ${ }^{1}$, E. Jehin ${ }^{2}$, D. Hutsemékers ${ }^{2}$, Y. Shinnaka ${ }^{3}$, J. Manfroid ${ }^{2}$, P. Rousselot ${ }^{4}$, S. Raghuram ${ }^{5}$, H. Kawakita ${ }^{3,6}$,
} A. Fitzsimmons ${ }^{7}$, K. Meech $^{8}$, M. Micheli ${ }^{9}$, C. Snodgrass ${ }^{1}$, B. Yang ${ }^{10}$, and O. Hainaut ${ }^{11}$

${ }^{1}$ Institute for Astronomy, University of Edinburgh, Royal Observatory, Edinburgh EH9 3HJ, UK e-mail: copi@roe.ac.uk

2 Space sciences, Technologies \& Astrophysics Research (STAR) Institute, University of Liège, Liège, Belgium

3 Koyama Astronomical Observatory, Kyoto Sangyo University, Motoyama, Kamigamo, Kita-ku, Kyoto 603-8555, Japan

4 Institut UTINAM UMR 6213, CNRS, Univ. Bourgogne Franche-Comté, OSU THETA, BP 1615, 25010 Besançon Cedex, France

${ }^{5}$ Laboratory for Atmospheric and Space Physics, University of Colorado Boulder, Boulder, CO, USA

${ }^{6}$ Department of Astrophysics and Atmospheric Sciences, Faculty of Science, Kyoto Sangyo University, Motoyama, Kamigamo, Kita-ku, Kyoto 603-8555, Japan

7 Astrophysics Research Centre, School of Mathematics and Physics, Queens University Belfast, Belfast BT7 1NN, UK

8 Institute for Astronomy, 2680 Woodlawn Drive, Honolulu, HI 96822, USA

9 ESA NEO Coordination Centre, Largo Galileo Galilei, 1, 00044 Frascati (RM), Italy

10 European Southern Observatory, Alonso de Cordova 3107, Vitacura, Santiago, Chile

11 European Southern Observatory, Karl-Schwarzschild-Strasse 2, 85748 Garching bei München, Germany

Received 4 May 2021 / Accepted 6 June 2021

\section{ABSTRACT}

\begin{abstract}
Aims. 2I/Borisov (hereafter 2I) is the first visibly active interstellar comet observed in the Solar System, allowing us for the first time to sample the composition of a building block from another system. We report on the monitoring of 2I with the Ultraviolet-Visual Echelle Spectrograph, the high-resolution optical spectrograph of the ESO Very Large Telescope at Paranal, for four months from November 15, 2019 to March 16, 2020. Our goal is to characterise the activity and composition of 2I with respect to Solar System comets.

Methods. We collected high-resolution spectra at 12 different epochs from 2.1 au pre-perihelion to 2.6 au post-perihelion.

Results. On December 24 and 26, 2019, close to perihelion, we detected several OH lines of the $309 \mathrm{~nm}(0-0)$ band and derived a water production rate of $2.2 \pm 0.2 \times 10^{26}$ molecules s$^{-1}$. The three [OI] forbidden oxygen lines were detected at different epochs and we derived a green-to-red doublet intensity ratio $(\mathrm{G} / \mathrm{R})$ of $0.31 \pm 0.05$ close to perihelion. The $\mathrm{NH}_{2}$ ortho and para lines from various bands were measured and allowed us to derive an ortho-to-para abundance ratio (OPR) of $3.21 \pm 0.15$, corresponding to an OPR and spin temperature of ammonia of $1.11 \pm 0.08$ and $31_{-5}^{+10} \mathrm{~K}$, respectively. These values are consistent with the values usually measured for Solar System comets. Emission lines of the radicals NH $(336 \mathrm{~nm}), \mathrm{CN}(388 \mathrm{~nm}), \mathrm{CH}(431 \mathrm{~nm})$, and $\mathrm{C}_{2}(517 \mathrm{~nm})$ were also detected. Several FeI and NiI lines were identified and their intensities were measured to provide a ratio of $\log (\mathrm{NiI} / \mathrm{FeI})=0.21 \pm 0.18$, which is in agreement with the value recently found in Solar System comets.

Conclusions. Our high spectral resolution observations of $2 \mathrm{I} / \mathrm{Borisov}$ and the associated measurements of the $\mathrm{NH}_{2} \mathrm{OPR}$ and the $\mathrm{Ni} / \mathrm{Fe}$ abundance ratio are remarkably similar to Solar System comets. Only the G/R ratio is unusually high, but it is consistent with the high abundance ratio of $\mathrm{CO} / \mathrm{H}_{2} \mathrm{O}$ found by other investigators.
\end{abstract}

Key words. comets: individual: 2I/Borisov - techniques: spectroscopic

\section{Introduction}

Interstellar objects provide an unprecedented opportunity to probe material that formed under potentially very different conditions from Solar System comets. 2I/Borisov (hereafter 2I) is only the second interstellar object to have been discovered and the first for which activity was directly detected. 2I was discovered in August 2019 at about 3 au from the Sun, prior to its perihelion passage, and it was already active at the time of discovery. 2I remained observable with major astronomical facilities for several months. This provided a unique opportunity to measure the composition of its coma and probe the ice composition of a comet that formed around another star.
Early observations, mainly with optical low-resolution spectrographs, showed that $2 \mathrm{I}$ is depleted in $\mathrm{C}_{2}$, similarly to Solar System carbon-chain depleted comets (Fitzsimmons et al. 2019; Opitom et al. 2019a; Bannister et al. 2020; Kareta et al. 2020; Lin et al. 2020; Aravind et al. 2021). Later, Cordiner et al. (2020) and Bodewits et al. (2020) reported that 2I is very rich in $\mathrm{CO}$ relative to both water and $\mathrm{HCN}$, more than any Solar System comet observed within $2.5 \mathrm{au}$ from the Sun. This provided the first indication that the composition of the first active interstellar comet might differ from that of most Solar System comets. Recent results indicated changes in the mixing ratio of species in the coma of 2I between pre- and post-perihelion observations, hinting at a heterogeneous nucleus (Xing et al. 2020; Yang et al. 2021; Aravind et al. 2021). 
We present here high-resolution spectroscopic observations of 2I performed at the Very Large Telescope, aimed at constraining the composition of the interstellar comet. In this work, we mainly focus on the gas component of the coma.

\section{Observation and data reduction}

We obtained 15 spectra of 2 I on 12 dates (for a total of $\sim 21$ hours) with the Ultraviolet-Visual Echelle Spectrograph (UVES) mounted on the ESO $8.2 \mathrm{~m}$ Very Large Telescope. We used three different settings to cover the full optical range: the dichroic\#1 $(346+580)$ setting covering the range from 303 to $388 \mathrm{~nm}$ in the blue and from 476 to $684 \mathrm{~nm}$ in the red; the free setting $(348+580)$ which is very similar to the $(346+580)$ setting, but for which the blue part is shifted by about $2 \mathrm{~nm}$ to the red; and the dichroic\#2 $(437+860)$ setting covering the range from 373 to $499 \mathrm{~nm}$ in the blue and from 660 to $1060 \mathrm{~nm}$ in the red. UVES has a slit length of $10^{\prime \prime}$ and we used slit widths of $0.4^{\prime \prime}, 0.7^{\prime \prime}$, and $1.8^{\prime \prime}$, providing resolving powers of about 80000,50000 , and 30000 , respectively. The observations were spread over four months between November 15, 2019 and March 16,2020 . Most of the observations analysed here were near perihelion on December 8, 2019. Very long exposures of up to $2 \mathrm{~h}$ were used to minimise the effect of the read-out noise for these observations of a faint target. The observing dates, set-ups, and observational circumstances are presented in Table 1.

The ESO UVES pipeline ${ }^{1}$ was used for the basic reduction, including wavelength calibration, extinction correction, and flux calibration using master response curves. We compared the flux calibration using master response curves and standard stars observed close to the science observations and found no significant differences. Since the UVES pipeline is not optimised for very extended objects, we used custom routines for the spectrum extraction over the full slit length and cosmic rays removal. The spectra were corrected for the Doppler shift due to the geocentric velocity of the comet. Finally, the dust continuum (as well as the moon and twilight contribution when necessary) were removed using a BASS2000 solar spectrum that was re-sampled to match the resolution of the observations in each setting. The BASS2000 was fitted individually for each component (dust-reflected sunlight, twilight, or moon contamination), allowing us to properly correct for the reddening of the comet continuum, for example. More details concerning the data reduction can be found in Manfroid et al. (2009).

\section{Analysis and results}

2I, with an $R$-band magnitude around 16.5 at perihelion (Jehin et al. 2020b), was relatively faint compared to comets usually observed at high spectroscopic resolution. In spite of that, emissions from several species were detected: $\mathrm{OH}, \mathrm{NH}$, $\mathrm{CN}, \mathrm{CH}, \mathrm{C}_{2}, \mathrm{NH}_{2}$, [OI], and metals FeI and NiI (see an extensive list in Table A.1). Unfortunately, none of the detected species had sufficiently strong emission bands to measure isotopic ratios. We searched for, but did not detect emissions from $\mathrm{N}_{2}^{+}, \mathrm{CO}^{+}$, $\mathrm{CO}_{2}^{+}, \mathrm{H}_{2} \mathrm{O}^{+}$, or $\mathrm{C}_{3}$.

\subsection{Nickel and iron abundances}

Manfroid et al. (2021) have recently shown, using UVES highresolution spectra collected over the last 20 years, that emis-

\footnotetext{
$1 \mathrm{ftp}$ ://ftp.eso.org/pub/dfs/pipelines/instruments/ uves/uves-pipeline-manual-6.1.3.pdf
}

sion lines of neutral $\mathrm{FeI}$ and $\mathrm{NiI}$ atoms are ubiquitous in the atmospheres of a number of Solar System comets of various composition, dynamical origin, and at different distances from the Sun (up to $3.2 \mathrm{au}$ ). Furthermore, NiI was detected in the coma of 2I by Guzik \& Drahus (2021). We searched our UVES spectra of 2I and identified several FeI and NiI lines (see Table A.1 and Fig. A.3). We followed the method described in Manfroid et al. (2021) and applied their multilevel atomic model, taking the true solar spectrum into account, to derive FeI and NiI production rates for 2I. We only used a subset of the lines listed in Table A.1 for this analysis, as some of these lines were of too low signal-to-noise ratio $(\mathrm{S} / \mathrm{N})$ or blended with other emission lines. The production rates were derived using an average spectrum from December 24 and 26, 2019 and a spectrum obtained on January 29, 2020, as these were the only spectra with a sufficient signal. Due to the different heliocentric velocities of the comet, the production rates for the two epochs were computed separately and then averaged, providing values of $\log \mathrm{Q}(\mathrm{NiI})=21.88 \pm 0.07$ molecules $\mathrm{s}^{-1}$ and $\log \mathrm{Q}(\mathrm{FeI})=21.67 \pm 0.16$ molecules s$^{-1}$, corresponding to $\log (\mathrm{NiI} / \mathrm{FeI})=0.21 \pm 0.18$. Our measurement of the NiI abundance is in very good agreement with the $9 \pm 3 \times$ $10^{21}$ molecules $^{-1}$ reported by Guzik \& Drahus (2021) for observations performed on January 28, 30, and 31, 2020.

Within the uncertainties, the $\log (\mathrm{NiI} / \mathrm{FeI})=0.21 \pm 0.18$ derived for $2 \mathrm{I}$ is in agreement with the average value of the ratio $\log (\mathrm{NiI} / \mathrm{FeI})=-0.06 \pm 0.31$ reported by Manfroid et al. (2021) for a sample of 17 Solar System comets (see Fig. 1). We also note that Manfroid et al. (2021) found a correlation between the total $\mathrm{Fe}$ and $\mathrm{Ni}$ production rates and water and $\mathrm{CO}$ production rates for most Solar System comets. We used our $\mathrm{Q}\left(\mathrm{H}_{2} \mathrm{O}\right)$ value of $2.2 \times 10^{26}$ molecules s ${ }^{-1}$ and a $\mathrm{Q}(\mathrm{CO})$ of $8 \times 10^{26}$ molecules s $^{-1}$ (interpolating between values for dates before and after our measurement from Bodewits et al. 2020) to estimate how 2I fits into these trends. While it fits within the trend for $\mathrm{Q}(\mathrm{CO})$ versus $\mathrm{Q}(\mathrm{Fe}+\mathrm{Ni}), 2 \mathrm{I}$ is an outlier for $\mathrm{Q}\left(\mathrm{H}_{2} \mathrm{O}\right)$ versus $\mathrm{Q}(\mathrm{Fe}+\mathrm{Ni})$, as illustrated in Figs. B.1 and B.2. Comet C/2016 R2 (PANSTARRS) is a Solar System comet extremely rich in CO (Biver et al. 2018), like 2I. Similar to C/2016 R2, 2I fits within the $\mathrm{Q}(\mathrm{CO})$ versus $\mathrm{Q}(\mathrm{Fe}+\mathrm{Ni})$ trend for Solar System comets, but not in the $\mathrm{Q}\left(\mathrm{H}_{2} \mathrm{O}\right)$ versus $\mathrm{Q}(\mathrm{Fe}+\mathrm{Ni})$ trend, strengthening the similarity between $2 \mathrm{I}$ and $\mathrm{C} / 2016 \mathrm{R} 2$.

\section{2. $\mathrm{NH}_{2}$ and $\mathrm{NH}_{3}$ ortho-to-para ratios}

We note that $\mathrm{NH}_{2}$ lines were detected in most of our spectra and used to measure the ortho-to-para abundance ratio (OPR) of $\mathrm{NH}_{2}$. The measurement of the OPR is of great interest, and even more in the case of an interstellar comet, as the relative abundance of different nuclear-spin isomers and the spin temperature that can be derived from it has been considered as an indicator of the temperature conditions prevailing when the molecules were formed, although this interpretation has been challenged recently (Hama et al. 2011).

We measured the $\mathrm{NH}_{2}$ OPR on four epochs in 2019, but the $\mathrm{S} / \mathrm{N}$ was unfortunately not sufficient for the 2020 observations. We used the two ro-vibronic emissions bands $(0,8,0)$ and $(0,9,0)$, following the method described in Shinnaka et al. (2011). For the $(0,8,0)$ band, we could determine the OPR separately for November 15, 2019 and for December 16, 24, and 26, 2019. For the $(0,9,0)$ band, the December 16,24 , and 26 observations had to be averaged to measure the $\mathrm{NH}_{2}$ OPR. The OPR of ammonia was computed considering $\mathrm{NH}_{3}$ as the sole parent of $\mathrm{NH}_{2}$, and keeping the total nuclear spin for the photodissociation 
Table 1. Observing circumstances of the 2I UVES campaign.

\begin{tabular}{lcccccccc}
\hline \hline Date (UT) & Instrument set-up & Slit width ${\left({ }^{\prime \prime}\right)}^{\prime}$ & $N$ & Exposure time $(\mathrm{s})$ & $r_{h}(\mathrm{au})$ & $\dot{r}_{h}\left(\mathrm{~km} \mathrm{~s}^{-1}\right)$ & $\Delta(\mathrm{au})$ & $\dot{\Delta}\left(\mathrm{km} \mathrm{s}^{-1}\right)$ \\
\hline $2019-11-15$ & $348+580$ & 0.7 & 1 & 3000 & 2.1 & -9.6 & 2.2 & -22.8 \\
$2019-12-16$ & $346+580$ & 1.8 & 1 & 3000 & 2.0 & 3.3 & 2.0 & -6.4 \\
$2019-12-24$ & $346+580$ & 1.8 & 1 & 6600 & 2.0 & 6.6 & 1.9 & -2.2 \\
$2019-12-25$ & $437+860$ & 1.8 & 1 & 6600 & 2.0 & 7.0 & 1.9 & -1.7 \\
$2019-12-26$ & $346+580$ & 1.8 & 1 & 6600 & 2.0 & 7.4 & 1.9 & -1.2 \\
$2020-01-29$ & $346+580$ & $0.4-1.8$ & 2 & $3350-6600$ & 2.3 & 18.9 & 2.1 & 11.5 \\
$2020-02-01$ & $346+580$ & 0.4 & 1 & 4500 & 2.3 & 19.7 & 2.1 & 12.2 \\
$2020-02-02$ & $346+580$ & 0.4 & 1 & 4500 & 2.4 & 19.9 & 2.1 & 12.4 \\
$2020-02-04$ & $346+580 / 437+860$ & 1.8 & 2 & 6600 & 2.4 & 20.4 & 2.1 & 12.9 \\
$2020-02-05$ & $346+580$ & 1.8 & 1 & 3000 & 2.4 & 20.7 & 2.1 & 13.2 \\
$2020-02-22$ & $348+580$ & 1.8 & 2 & $2870-4875$ & 2.6 & 24.2 & 2.3 & 16.0 \\
$2020-03-16$ & $348+580$ & 1.8 & 1 & 6600 & 2.6 & 24.2 & 2.3 & 16.0 \\
\hline
\end{tabular}

Notes. $N$ represents the number of exposures obtained.

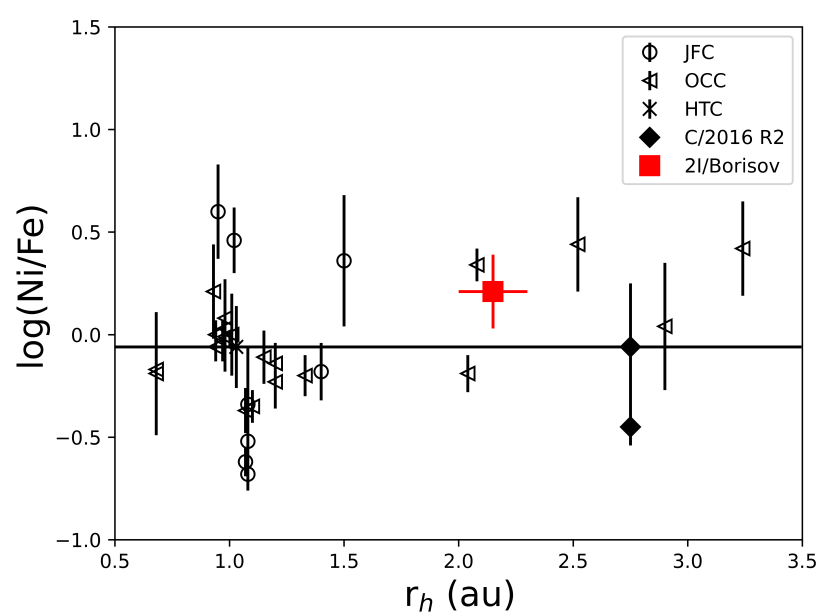

Fig. 1. Comparison between the $\log (\mathrm{Ni} / \mathrm{Fe})$ ratio of $2 \mathrm{I}$ and the values measured by Manfroid et al. (2021) for a sample of Solar System comets (Jupiter-family comets (JFC), Oort cloud comets (OCC), and Halley-type comets (HTC)). The horizontal line represents the average value for Solar System comets from Manfroid et al. (2021).

reaction. Our measurements are listed for each band in Table 2. The weighted average $\mathrm{NH}_{2}$ OPR value for both bands is $3.21 \pm 0.15$, corresponding to a $\mathrm{NH}_{3}$ OPR of $1.11 \pm 0.08$. A nuclear spin temperature $\left(T_{\text {Spin }}\right)$ for ammonia of $31_{-5}^{+10} \mathrm{~K}$ was derived. Within the uncertainties, we do not see an evolution of the OPR with time or heliocentric distance, consistent with what is usually observed for Solar System comets.

2I's $\mathrm{NH}_{2} \mathrm{OPR}$ (and corresponding $\mathrm{NH}_{3} \mathrm{OPR}$ and spin temperature) are remarkably similar to the typical values measured in Solar System comets (see Fig. 2). The ammonia OPR and spin temperature have been thought to be linked to the formation temperature of the molecule. If this is the case, the similar OPR and spin temperature values would indicate that 2I had a similar formation environment to our own Solar System. However, recent laboratory experiments have shown that the water OPR might not retain the memory of the molecule formation temperature (Hama et al. 2011, 2016; Hama \& Watanabe 2013), but rather be diagnostic of the physico-chemical conditions in the innermost coma or in the subsurface layers. If this is verified, a similar argument could be made for the ammonia OPR and spin temperature. It is thus likely that the similar $\mathrm{NH}_{2}$ OPR
Table 2. $\mathrm{NH}_{2}$ and $\mathrm{NH}_{3}$ OPRs and spin temperatures measured in the coma of 2I.

\begin{tabular}{lccc}
\hline \hline Date & $r_{h}$ & $\mathrm{NH}_{2} \mathrm{OPR}$ & $\mathrm{NH}_{3}$ \\
\hline \multicolumn{4}{c}{$\mathrm{NH}_{2}(0,8,0)$ band } \\
\hline $2019-11-15$ & 2.1 & $3.07 \pm 0.23$ & $1.04 \pm 0.12$ \\
$2019-12-16$ & 2.0 & $3.09 \pm 1.07$ & $1.05 \pm 0.54$ \\
$2019-12-24$ & 2.0 & $3.29 \pm 0.36$ & $1.15 \pm 0.18$ \\
$2019-12-26$ & 2.0 & $3.89 \pm 0.88$ & $1.45 \pm 0.44$ \\
\hline Average & $3.17 \pm 0.19$ \\
$T_{\text {Spin }}$ & $>27(1 \sigma) \mathrm{K}$ \\
$T_{\text {Spin }}$ & $>22(3 \sigma) \mathrm{K}$ \\
\hline \multicolumn{4}{c}{} \\
\hline 2019-11-15 & $\mathrm{NH}_{2}(0,9,0)$ band \\
$2019-12-16,24,26$ & 2.1 & $3.63 \pm 0.38$ & $1.32 \pm 0.19$ \\
Average & $2.99 \pm 0.39$ & $1.00 \pm 0.20$ \\
$T_{\text {Spin }}$ & $3.32 \pm 0.27$ \\
\hline
\end{tabular}

for 2I and Solar System comets simply reflect similar physicochemical conditions in the inner coma.

\subsection{Water production rate}

As mentioned before, we detected the $\mathrm{OH}(0-0)$ band around $309 \mathrm{~nm}$ in our spectra, from which we computed the production rate of water. We used spectra obtained on December 24 and 26, about two weeks after the comet's perihelion passage, as these were the ones with the best $\mathrm{S} / \mathrm{N}$. To further increase the $\mathrm{S} / \mathrm{N}$, we averaged the two spectra. We used a Haser model to compute the production rate, with scalelengths from Cochran \& Schleicher (1993) for OH, and our own model of $\mathrm{OH}$ fluorescence spectrum (Rousselot et al. 2019) for computing the fluorescence efficiency. This model provided a fluorescence efficiency of $6.0 \times 10^{-16} \mathrm{erg}$. $\mathrm{s}^{-1}$. molecule ${ }^{-1}$ with the average heliocentric distance and velocity of 2 I corresponding to the two spectra $\left(2.04 \mathrm{au}\right.$ and $+7.02 \mathrm{~km} . \mathrm{s}^{-1}$, respectively). Such a value can be compared, for example, to the fluorescence model published by Schleicher \& A'Hearn (1988), which would provide about $6.5 \times 10^{-16}$ erg.s ${ }^{-1}$.molecule ${ }^{-1}$ for similar parameters. This small difference could be due to different parameters used for both models (e.g., transition probabilities, solar atlas). We assumed an equal velocity for the daughter and parent of 


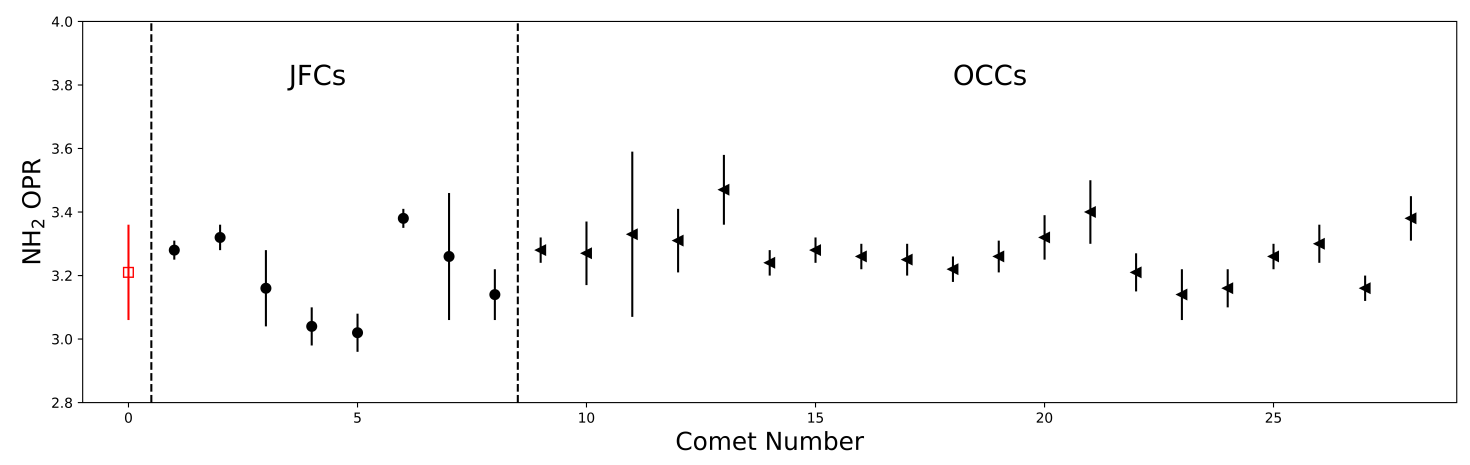

Fig. 2. $\mathrm{NH}_{2}$ OPR of $28 \mathrm{JFCs}$ and OCCs from the Solar System (black symbols), compared to the value measured for the interstellar comet $2 \mathrm{I}$ (red square). Adapted from Shinnaka et al. (2016), with additional data from Yang et al. (2019) and Shinnaka et al. (2020).

$595 \mathrm{~m} \mathrm{~s}^{-1}$, following the formula $\mathrm{v}\left(\mathrm{H}_{2} \mathrm{O}\right)=850 / \sqrt{r_{h}} \mathrm{~m} \mathrm{~s}^{-1}$ given in Cochran \& Schleicher (1993). Given the low $\mathrm{S} / \mathrm{N}$ around $309 \mathrm{~nm}$, we summed the seventeen brightest apparent lines (corresponding to 27 real lines, some of them being blended) and measured the corresponding total flux equal to $4.5 \pm 0.5 \times$ $10^{-15}$ erg.s ${ }^{-1} . \mathrm{cm}^{-2}$ for the $1.8^{\prime \prime}$-wide slit (see Fig. 3). The final total intensity is sensitive to different parameters, such as errors in offset and baseline corrections. The final spectrum allowed us to estimate these errors to about $0.5 \times 10^{-15} \mathrm{erg} . \mathrm{s}^{-1} . \mathrm{cm}^{-2}$, with the average baseline being very close to zero in intensity. With the parameters given above, we computed a produc-

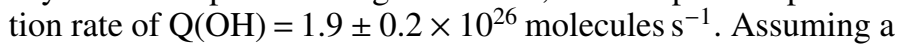
ratio of the production rate of $\mathrm{OH}$ radicals to the total photodestruction rate of water (photodissociation plus ionisation) for a quiet Sun (corresponding to the time of observations) of $87 \%$ (Crovisier 1989), this corresponds to a water production rate of $\mathrm{Q}\left(\mathrm{H}_{2} \mathrm{O}\right)=2.2 \pm 0.2 \times 10^{26}$ molecules s $^{-1}$.

A water production rate from UVES spectra was already reported by Jehin et al. (2020a), and it is consistent with what we find in this work after improving the flux calibration. Water production rates were also measured by Xing et al. (2020) between November 1 and December 21, 2019. On December 21, they measured $\mathrm{Q}\left(\mathrm{H}_{2} \mathrm{O}\right)=4.9 \pm 0.9 \times 10^{26}$ molecules $\mathrm{s}^{-1}$. This is more than two times higher than the value we measured only a few days later. However, we note that Xing et al. (2020) used a vectorial model to derive the water production rate, while we used a Haser model. Re-computing the water production rate with the same model and model parameters, we found $\mathrm{Q}\left(\mathrm{H}_{2} \mathrm{O}\right)=3.4 \times 10^{26}$ molecules $\mathrm{s}^{-1}$. Considering the steep postperihelion decrease in the water production rate reported by Xing et al. (2020), this value is consistent with their measurements and confirms the decreasing trend.

\subsection{Forbidden oxygen emission lines}

As shown in Table A.1, we detected forbidden oxygen lines at 557.73, 630.03, and $636.38 \mathrm{~nm}$ in the coma of 2I. Atomic oxygen atoms in the coma of comets are produced by the photo-dissociation of oxygen-bearing species, the most abundant contributors being $\mathrm{H}_{2} \mathrm{O}, \mathrm{CO}, \mathrm{CO}_{2}$, and $\mathrm{O}_{2}$. It has been shown that the ratio between the intensity of the green oxygen line (at $557.73 \mathrm{~nm}$ ) and the red-doublet (at 630.03 and $636.38 \mathrm{~nm}$ ) depends on the relative abundance of the different parents in the coma (Festou \& Feldman 1981; McKay et al. 2013; Decock et al. 2013, 2015). This ratio is usually referred to as the $\mathrm{G} / \mathrm{R}$ ratio. The cometary forbidden oxygen lines could only be resolved from their telluric counterparts and the $G / R$ ratio measured from some spectra. On November 15, 2019, we

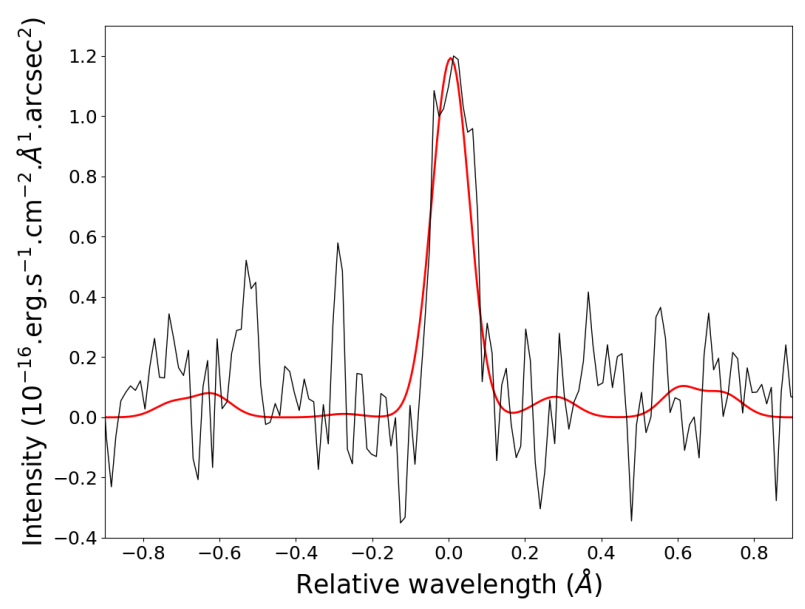

Fig. 3. Average of the 17 brightest $\mathrm{OH}$ lines in the average spectrum of comet 2I obtained on December 24 and 26, 2019 with UVES at the VLT. The red line represents the fitted fluorescence model.

measured a $\mathrm{G} / \mathrm{R}=0.31 \pm 0.05$, while the comet was at 2.1 au from the Sun pre-perihelion. We combined the spectra from January 29, February 1, and February 2, 2020 to increase the signal-tonoise ratio and found a $\mathrm{G} / \mathrm{R}=0.3 \pm 0.1$ (at $r_{h}=2.3 \mathrm{au}$ ). We also combined the data from February 22 and March 16 to better constrain its evolution with time and heliocentric distance and measured $\mathrm{G} / \mathrm{R}=0.6 \pm 0.3$. More details about the measurement of the $\mathrm{G} / \mathrm{R}$ ratio can be found in Appendix $\mathrm{C}$.

These values are high compared to what is usually measured in comets at the same distance from the Sun, as illustrated in Fig. 4. For a sample of 11 comets, Decock et al. (2013) report a mean value of $0.11 \pm 0.07$. It has been observed by both Decock et al. (2013) and McKay et al. (2013) that the values of the $\mathrm{G} / \mathrm{R}$ ratio increase for comets observed at large heliocentric distances, but this increase of the $\mathrm{G} / \mathrm{R}$ ratio is usually noticed for comets beyond 2.5 au (Decock et al. 2013). The only other outlier in Fig. 4 is C/2016 R2, which similar to 2I was very rich in $\mathrm{CO}$. The $\mathrm{G} / \mathrm{R}$ ratio expected if the oxygen atoms are produced from the photo-dissociation of water is around 0.08 , but it is much higher (around 0.78) if they are produced by the photodissociation of CO (Bhardwaj \& Raghuram 2012). The higher than usual $\mathrm{G} / \mathrm{R}$ ratio we measured for $2 \mathrm{I}$ might thus be the consequence of its high $\mathrm{CO} / \mathrm{H}_{2} \mathrm{O}$ ratio. As the heliocentric distance increases, we would expect the decrease in the water production rate to cause a decrease in the intensity of the red-doublet. At the same time, the intensity of the green line, to which the CO contributes significantly, would not decrease as much. As a 


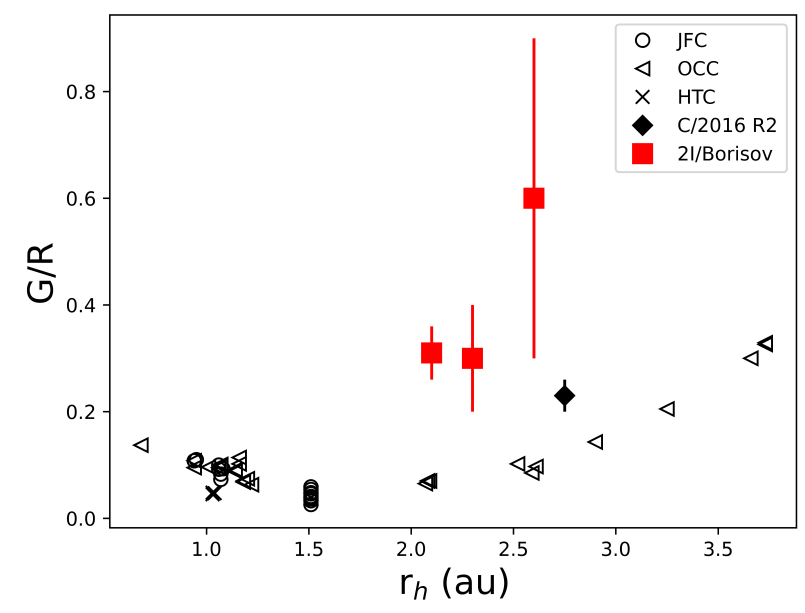

Fig. 4. Ratio between the green and the sum of the two red forbidden oxygen lines $(\mathrm{G} / \mathrm{R})$ in 2I, compared to that measured for a sample of Solar System comets by Decock et al. (2013) (and Opitom et al. 2019b for $\mathrm{C} / 2016 \mathrm{R} 2$ ).

consequence, the $\mathrm{G} / \mathrm{R}$ ratio would increase, which could explain the higher $\mathrm{G} / \mathrm{R}$ ratio measured in March. Dedicated chemistryemission modelling is out of the scope of this work, but it will be explored in the future to further investigate the cause of the high $\mathrm{G} / \mathrm{R}$ ratio of $2 \mathrm{I}$.

\section{Conclusions and perspectives}

We observed the first active interstellar comet 2I/Borisov with the UVES high-resolution spectrograph on 12 dates between November 15, 2019 and March 16, 2020. These observations constitute a unique data set to constrain the composition of the interstellar visitor. Using these spectra, we performed various measurements, some being made for the first time in an interstellar comet.

In particular, we measured a NiI to FeI abundance ratio consistent with what has been found for a sample of 17 Solar System comets by Manfroid et al. (2021). In the future, the Ni/Fe ratio measured in the coma of interstellar comets could complement molecular abundances already used and provide an additional tool to probe the composition of interstellar comets' native systems. Indeed, the relative abundances of metals produced by type 1a supernovae nucleosynthesis varies depending on the explosion mechanism or the initial conditions of the white dwarf (Leung \& Nomoto 2018; Palla 2021). The material produced by those supernovae then feeds the interstellar medium and can be integrated in forming planetary systems, leaving an imprint on the composition of comets that could be measurable given the large variations of the $\mathrm{Ni} / \mathrm{Fe}$ ratios observed in supernovae (Mori et al. 2018).

We also derived the $\mathrm{NH}_{2}$ OPR (and corresponding $\mathrm{NH}_{3} \mathrm{OPR}$ and spin temperature) of $2 \mathrm{I}$ and find them similar to Solar System comets. This likely reflects similar physico-chemical conditions in the inner coma. We detected forbidden oxygen lines and computed $\mathrm{G} / \mathrm{R}$ ratios higher than what is usually found for Solar System comets at that distance from the Sun. However, this is consistent with the high $\mathrm{CO}$ abundance in the coma of $2 \mathrm{I}$ and the increase of the $\mathrm{CO} / \mathrm{H}_{2} \mathrm{O}$ ratio with the heliocentric distance. In conclusion, our high spectral resolution observations of 2I/Borisov reveal a remarkable similarity to Solar System comets in terms of OPR and $\mathrm{Ni} / \mathrm{Fe}$ abundances. The $\mathrm{G} / \mathrm{R}$ ratio is high, similar to $\mathrm{C} / 2016 \mathrm{R} 2$, confirming a high $\mathrm{CO} / \mathrm{H}_{2} \mathrm{O}$ abundance ratio, and suggesting that both comets might have formed in colder environments.

Acknowledgements. Based on observations collected at the European Southern Observatory under ESO programmes 2103.C-5068(F) and 105.205Q.006. We are grateful to the ESO Paranal staff and user support department for their efforts and exceptional support in obtaining the observations. C. O. is a Royal Astronomical Society Norman Lockyer fellow and University of Edinburgh Chancellor's fellow. J. M., D. H., and E. J. are honorary Research Director, Research Director and Senior Research Associate at the F.R.S-FNRS, respectively.

\section{References}

Aravind, K., Ganesh, S., Venkataramani, K., et al. 2021, MNRAS, 502, 3491 Bannister, M. T., Opitom, C., Fitzsimmons, A., et al. 2020, AAS J., submitted [arXiv:2001.11605]

Bhardwaj, A., \& Raghuram, S. 2012, ApJ, 748, 13

Biver, N., Bockelée-Morvan, D., Paubert, G., et al. 2018, A\&A, 619, A127

Bodewits, D., Noonan, J. W., Feldman, P. D., et al. 2020, Nat. Astron., 4, 867

Cochran, A. L., \& Schleicher, D. G. 1993, Icarus, 105, 235

Cordiner, M. A., Milam, S. N., Biver, N., et al. 2020, Nat. Astron., 4, 861

Crovisier, J. 1989, A\&A, 213, 459

Decock, A., Jehin, E., Hutsemékers, D., \& Manfroid, J. 2013, A\&A, 555, A34

Decock, A., Jehin, E., Rousselot, P., et al. 2015, A\&A, 573, A1

Festou, M., \& Feldman, P. D. 1981, A\&A, 103, 154

Fitzsimmons, A., Hainaut, O., Meech, K. J., et al. 2019, ApJ, 885, L9

Guzik, P., \& Drahus, M. 2021, Nature, 593, 375

Hama, T., \& Watanabe, N. 2013, Chem. Rev., 113, 8783

Hama, T., Watanabe, N., Kouchi, A., \& Yokoyama, M. 2011, ApJ, 738, L15

Hama, T., Kouchi, A., \& Watanabe, N. 2016, Science, 351, 65

Jehin, E., Yang, B., Hainaut, O., et al. 2020a, in European Planetary Science Congress, EPSC2020-653

Jehin, E., Manfroid, J., Hutsemekers, D., et al. 2020b, COMET 2I/2019 Q4 (BORISOV), Cent. Bureau Electron. Telegraph 4719

Kareta, T., Andrews, J., Noonan, J. W., et al. 2020, ApJ, 889, L38

Leung, S.-C., \& Nomoto, K. 2018, ApJ, 861, 143

Lin, H. W., Lee, C.-H., Gerdes, D. W., et al. 2020, ApJ, 889, L30

Manfroid, J., Jehin, E., Hutsemékers, D., et al. 2009, A\&A, 503, 613

Manfroid, J., Hutsemékers, D., \& Jehin, E. 2021, Nature, 593, 372

McKay, A. J., Chanover, N. J., Morgenthaler, J. P., et al. 2013, Icarus, 222, 684

McKay, A. J., Cochran, A. L., Dello Russo, N., \& DiSanti, M. A. 2020, ApJ, 889, L10

Mori, K., Famiano, M. A., Kajino, T., et al. 2018, ApJ, 863, 176

Opitom, C., Fitzsimmons, A., Jehin, E., et al. 2019a, A\&A, 631, L8

Opitom, C., Hutsemékers, D., Jehin, E., et al. 2019b, A\&A, 624, A64 Palla, M. 2021, MNRAS, 503, 3216

Rousselot, P., Opitom, C., Jehin, E., et al. 2019, A\&A, 628, A22

Schleicher, D. G., \& A'Hearn, M. F. 1988, ApJ, 331, 1058

Shinnaka, Y., Kawakita, H., Kobayashi, H., et al. 2011, ApJ, 729, 81

Shinnaka, Y., Kawakita, H., Jehin, E., et al. 2016, MNRAS, 462, S124

Shinnaka, Y., Kawakita, H., \& Tajitsu, A. 2020, AJ, 159, 203

Xing, Z., Bodewits, D., Noonan, J., \& Bannister, M. T. 2020, ApJ, 893, L48

Yang, B., Jehin, E., Pozuelos, F. J., et al. 2019, A\&A, 631, A168

Yang, B., Li, A., Cordiner, M. A., et al. 2021, Nat. Astron., 5, 586 


\section{Appendix A: Detected species}

Table A.1. Species detected in the coma of 2I from UVES observations.

\begin{tabular}{|c|c|}
\hline Species & Detected band/lines \\
\hline $\mathrm{OH}$ & $(0,0)[310 \mathrm{~nm}] \mathrm{A}^{2} \Sigma^{+}-\mathrm{X}^{2} \Pi$ \\
\hline $\mathrm{NH}$ & $\mathrm{A}^{3} \Pi_{\mathrm{i}}-\mathrm{X}^{3} \Sigma^{-}[336 \mathrm{~nm}]$ \\
\hline $\mathrm{CN}$ & $(0,0)[388 \mathrm{~nm}],(1,0)[422 \mathrm{~nm}] \mathrm{B}^{2} \Sigma^{+}-\mathrm{X}^{2} \Sigma^{+}$ \\
\hline $\mathrm{CH}$ & $(0,0)[431 \mathrm{~nm}] \mathrm{A}^{2} \Delta-\mathrm{X}^{2} \Pi$ \\
\hline $\mathrm{C}_{2}$ & $(0,0)[517 \mathrm{~nm}] \mathrm{d}^{3} \Pi_{g}-\mathrm{A}^{3} \Pi_{u}$ Swan System \\
\hline $\mathrm{NH}_{2}$ & $\begin{array}{l}(0,12,0)[520 \mathrm{~nm}],(1,7,0)[540 \mathrm{~nm}],(0,11,0)[543 \mathrm{~nm}],(0,10,0)[570 \mathrm{~nm}],(0,9,0)[600 \mathrm{~nm}],(0,8,0)[630 \mathrm{~nm}], \\
(0,7,0)[665 \mathrm{~nm}],(0,6,0)[695 \mathrm{~nm}] \mathrm{A}-\mathrm{X}\end{array}$ \\
\hline [OI] & $557.73,630.03$, and $636.37 \mathrm{~nm}$ \\
\hline NiI & $336.96,339.30,341.48,343.36,344.63^{(*)}, 345.85,346.17,349.30^{(*)}, 351.03,351.51,352.45,356.64^{(*)}, 361.94 \mathrm{~nm}$ \\
\hline FeI & $344.06,349.06,358.12,371.99,373.49^{(*)}, 374.56,374.59,374.83,374.95,375.82 \mathrm{~nm}$ \\
\hline
\end{tabular}

Notes. The blended FeI and NiI lines are marked by ${ }^{(*)}$. Non detected species: $\mathrm{N}_{2}^{+}, \mathrm{CO}^{+}, \mathrm{CO}_{2}^{+}, \mathrm{H}_{2} \mathrm{O}^{+}$, and $\mathrm{C}_{3}$. The number between brackets represent the approximate wavelength of the emission band.

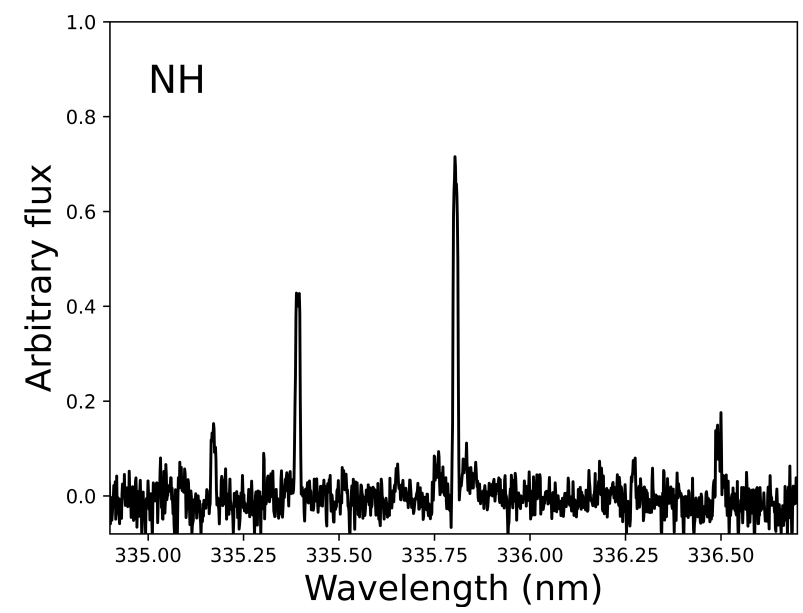

Fig. A.1. $\mathrm{NH} \mathrm{A}^{3} \Pi_{\mathrm{i}}-\mathrm{X}^{3} \Sigma^{-}$band in the coma of $2 \mathrm{I} /$ Borisov around $335 \mathrm{~nm}$.

In this appendix, we give the details of the species and specific emission bands detected in our high-resolution spectra of 2I. Table A.1 summarises our detections and non-detections. In addition to the $\mathrm{NH}, \mathrm{CN}, \mathrm{C}_{2}$, and $\mathrm{NH}_{2}$ emission bands, the $630 \mathrm{~nm}[\mathrm{OI}]$ line, and neutral nickel emission lines that were detected in previous observations (Fitzsimmons et al. 2019; Bannister et al. 2020; Aravind et al. 2021; Kareta et al. 2020; McKay et al. 2020; Guzik \& Drahus 2021), we report the detection of a $\mathrm{CH}$ emission band, 557.7 and $636.4 \mathrm{~nm}$ forbidden oxygen lines, as well as emission lines from FeI. Our detection of $\mathrm{NH}, \mathrm{CH}, \mathrm{FeI}$, and NiI emissions are illustrated in Figs. A.1-A.3, respectively.

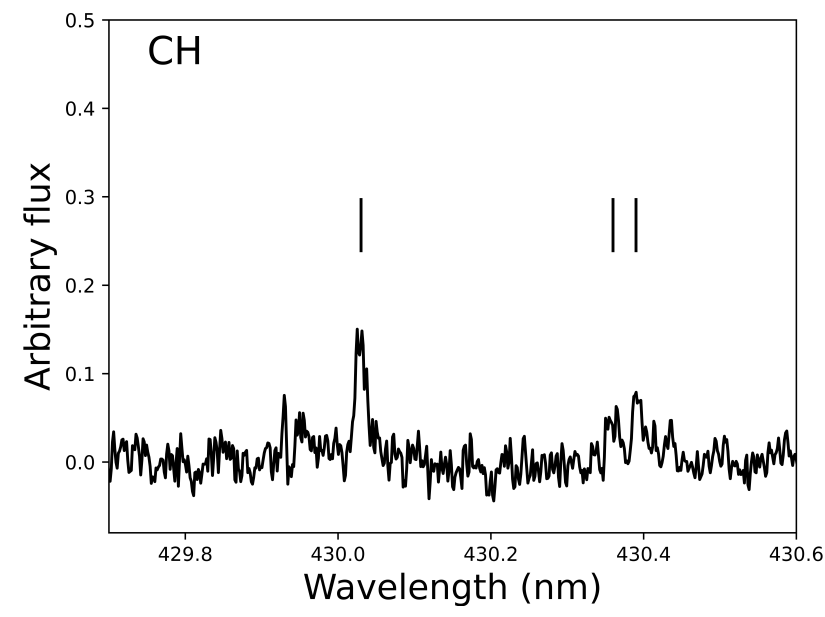

Fig. A.2. Spectrum of $2 \mathrm{I}$ over the $429.7-430.6 \mathrm{~nm}$ range covering three prominent $\mathrm{CH}$ lines from the $(0,0) \mathrm{A}^{2} \Delta-\mathrm{X}^{2} \Pi$ band (indicated by vertical black lines).

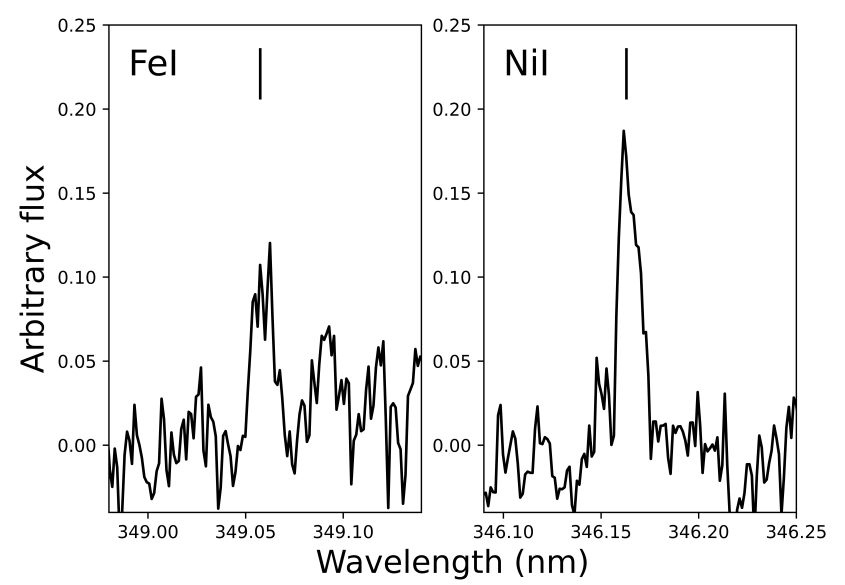

Fig. A.3. Example of FeI and NiI lines detected in the coma of $2 \mathrm{I}$ at 349.06 and $346.17 \mathrm{~nm}$, respectively. 
Appendix B: Correlation between Fel, $\mathrm{Nil}, \mathrm{H}_{2} \mathrm{O}$, and $\mathrm{CO}$

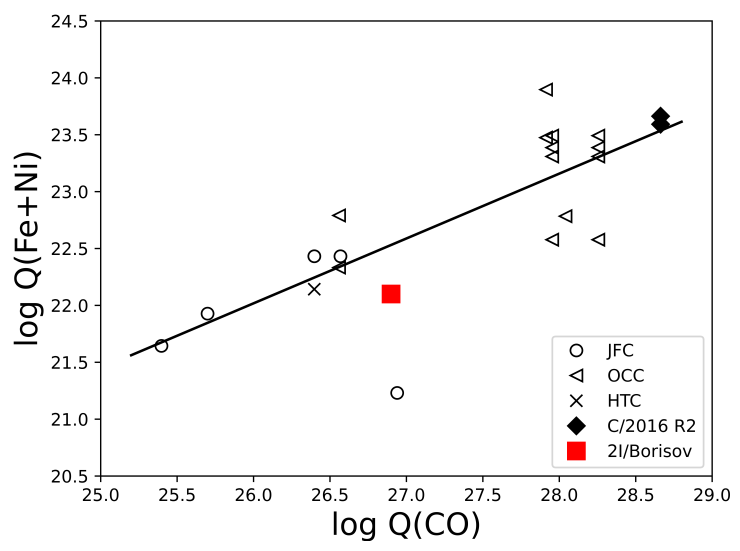

Fig. B.1. Sum of the production rates of FeI and NiI as a function of the $\mathrm{CO}$ production rate for $2 \mathrm{I} /$ Borisov, compared to the values measured by Manfroid et al. (2021) for a sample of Solar System comets (JFC, OCC, and HTC). The black line represents the best correlation for Solar System comets from Manfroid et al. (2021) (excluding C/2016 R2). The JFC located below the trend is 9P/Tempel 1 .

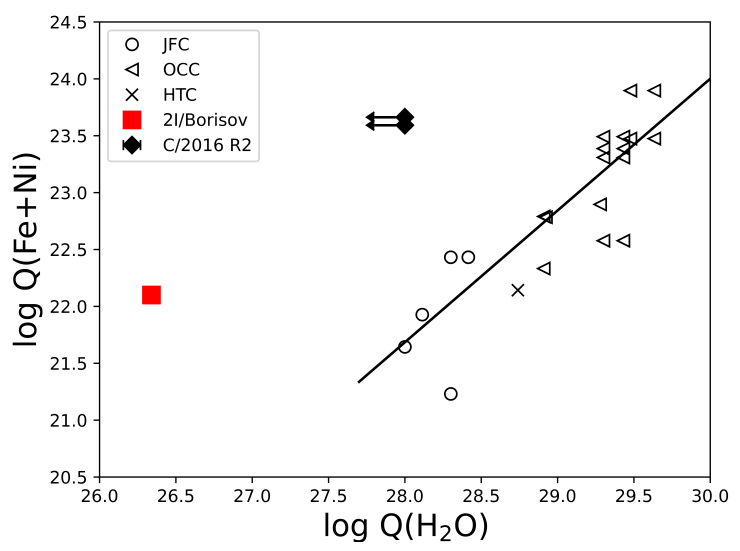

Fig. B.2. Sum of the production rates of FeI and NiI as a function of the water production rate for $2 \mathrm{I} /$ Borisov, compared to the values measured by Manfroid et al. (2021) for a sample of Solar System comets (JFC, OCC, and HTC). The black line represents the best correlation for Solar System comets from Manfroid et al. (2021) (excluding C/2016 R2).

In Sect. 3.1, we discuss correlations between the total $\mathrm{Fe}$ and $\mathrm{Ni}$ production rate with the $\mathrm{CO}$ and the water production rates in $2 \mathrm{I}$ compared to Solar System comets measured by Manfroid et al. (2021). In Fig. B.1, which represents the total FeI and NiI production rate as a function of the $\mathrm{CO}$ production rate, 2I fits well within the trend defined by Solar System comets. In Fig. B.2, however, 2I is outside the trend, indicating a lower water production rate compared to the total $\mathrm{Fe}$ and Ni production. The only other outlier is C/2016 R2 (PANSTARRS), which also does not fit with the trend defined by other Solar System comets, reinforcing the similarities between 2I and C/2016 R2.

\section{Appendix C: Determination of the $\mathrm{G} / \mathrm{R}$ ratio}

We were able to measure the $\mathrm{G} / \mathrm{R}$ ratio at three different epochs. For each epoch, we modelled the cometary and telluric components of the forbidden oxygen lines simultaneously. For November 15,2019 , the $0.7^{\prime \prime}$ slit combined with the comet geocentric velocity of $-22.8 \mathrm{~km} \mathrm{~s}^{-1}$ allowed us to resolve the cometary and telluric components. We used a single Gaussian to model each line and our best fit is shown in Fig. C.1a. We then integrated the flux for the best-fit model of the cometary lines and computed the ratio between the intensity of the green line at $557.73 \mathrm{~nm}$ and the sum of the two red ones at 630.03 and $636.37 \mathrm{~nm}$ to obtain a $\mathrm{G} / \mathrm{R}=0.31 \pm 0.05$. For the first two epochs, we determined that the main source of uncertainty in the measurement of the $G / R$ ratio was the subtraction of the dust continuum. We thus estimated the uncertainties by varying the level of the continuum at the wavelength of the oxygen lines prior to the subtraction by $\pm 10 \%$.

For the second epoch, we averaged three spectra from January 29, February 1, and February 2 2020, all obtained with a $0.4^{\prime \prime}$ slit. The geocentric velocity of the comet at that time was lower, which resulted in a minor blend of the telluric and cometary lines, especially for the green line. As can be seen in Fig. C.1b, the $557 \mathrm{~nm}$ telluric line (left panel) has wide wings that affect the measurement of the cometary line. We thus used a sum of two Gaussians to represent the telluric [OI] line around $557.73 \mathrm{~nm}$. All other lines could be satisfactorily reproduced by simple Gaussians. Due to the blend, we could not fit the width of the green cometary line and fixed it as the same as the red $630.03 \mathrm{~nm}$ line. Tests varying the width of the green line confirmed that the change in the value of the $G / R$ ratio is within the uncertainty of the measurement. The best fit is represented in Fig. C. $1 b$ and resulted in $\mathrm{G} / \mathrm{R}=0.3 \pm 0.1$.

For the last epoch, we used spectra from February 22 and March 16, obtained with a wide slit of $1.8^{\prime \prime}$, which resulted in a blend of the telluric and cometary lines. Fortunately, even for the green line, we still see a knee in the telluric line corresponding to the comet emission. Because such a large slit was used, the telluric lines were not well reproduced by a Gaussian. To better match the shape of the telluric lines, we convolved the Gaussian with a box function. We added one Gaussian for the $636 \mathrm{~nm}$ line and two for the $557 \mathrm{~nm}$ line to satisfactorily reproduce the wings of the telluric lines. In this case, we had to manually vary the fit parameters to reach the best fit shown in Fig. C.1c, corresponding to $\mathrm{G} / \mathrm{R}=0.6 \pm 0.3$. We estimated the uncertainty by varying the parameters of the model and computing the difference in the $\mathrm{G} / \mathrm{R}$ ratio value between the most extreme fits that provided a satisfactory representation of the data. Because of the difficulty of de-blending the cometary and telluric line and the high uncertainty it causes on the measurement of the $\mathrm{G} / \mathrm{R}$ ratio, we consider this measurement as tentative. 


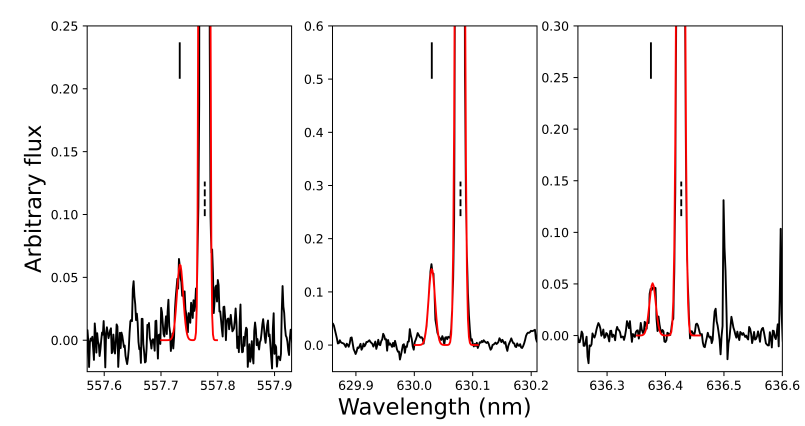

(a)

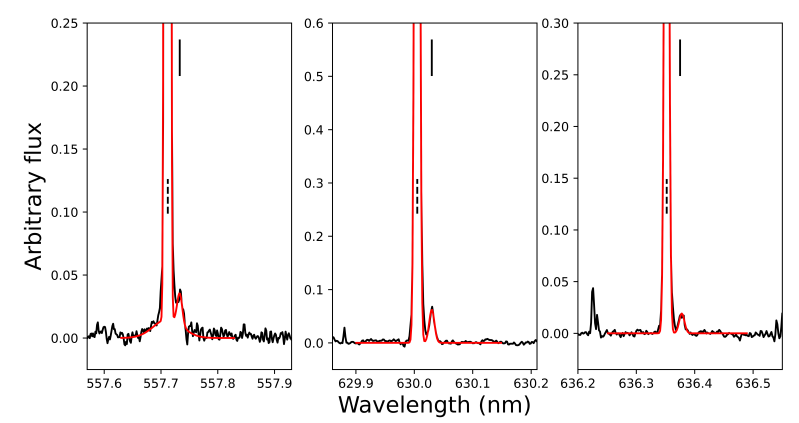

(b)

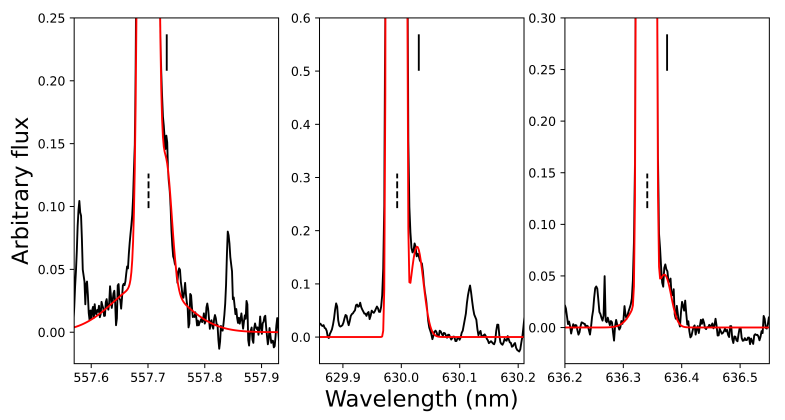

(c)

Fig. C.1. Spectra of 2I around the 557.73 (left), 630.03 (centre), and $636.37 \mathrm{~nm}$ (right) forbidden oxygen lines at three different epochs (black line). Our best fit model, used to measure the G/R ratio, is represented in red. The black vertical mark indicates the position of the cometary line and the dashed mark indicates the position of the telluric line. (a) November 15, 2019. (b) January 29, February 1, and February 2, 2020. (c) February 22 and March 16, 2020. 